\title{
Computational Approach to Structural Analysis of Protein-RNA Complexes*
}

\author{
Namshik Han, Hyunwoo Kim, and Kyungsook Han ${ }^{*}$ \\ School of Computer Science and Engineering, Inha University, Inchon 402-751, Korea \\ han_3567@hotmail.com, whytok@hanmail.net, khan@inha.ac.kr
}

\begin{abstract}
Analyzing protein-RNA binding structures depends on a significant quantity of manual work. Therefore, the protein-RNA binding structures are generally studied individually or on a small-scale. The task of analyzing the protein-RNA binding structures manually becomes increasingly difficult as the complexity and number of protein-RNA binding structures increase. We have developed a set of algorithms for automatically analyzing the structures of the protein-RNA complexes at an atomic level and for identifying the interaction patterns between the protein and RNA. The algorithms were implemented and tested on the actual structure data of 51 protein-RNA complexes. It is believed that this is the first structural analysis of a large set of protein-RNA complexes. The results of the analysis will provide insight into the interaction patterns between a protein and RNA, and will be useful in predicting the structure of the RNA binding protein and the structure of the protein binding RNA.
\end{abstract}

\section{Introduction}

Identifying how a protein molecule binds an RNA molecule with an affinity and specificity is a complex problem involving both spatial reasoning and an extensive knowledge of biochemistry. Individual or a small set of protein-RNA binding structures can be analyzed manually. However, as the number of protein-RNA complexes available in public databases is rapidly increasing a more systematic and automated method will be needed.

In contrast to the regular helical structure of DNA, RNA molecules form complex secondary and tertiary structures consisting of several structure elements, including stems, loops, and pseudoknots. Often, only specific proteins can recognize the structure elements arranged three-dimensional space. RNA structures display hydrogen bonding, electrostatic, and hydrophobic groups that can interact with small molecules to form specific contacts. However, it is unclear how the proteins interact with the RNA with specificity.

This paper presents a computational approach to analyze the interactions between the amino acids of a protein and the RNA nucleotides at the atomic level. The primary

\footnotetext{
* This work was supported by the Ministry of Information and Communication of Korea under grant number 01-PJ11-PG9-01BT00B-0012.

** To whom correspondence should be addressed.
} 
focus of the work was to discover the conspicuous preferences in the pairing of amino acids with nucleotides. We have developed a set of algorithms for analyzing the protein-RNA binding structures and tested the algorithms on 51 protein-RNA complexes obtained from the PDB database [4]. The computational analysis attempted to address the following problems: (1) the hydrogen bonding propensity between the amino acids and the nucleotides, (2) the preferences between the main and side protein chains in the binding sites, and (3) the preferences between the base and RNA backbone in the binding sites.

\section{Terminology and Notation}

\subsection{The Amino Acids: Main Chain, Side Chain}

Proteins are sequences of up to 20 types of amino acids. Each amino acid contains the main chain of repeating units and one of 20 different " $R$ " groups. It is the structure of the R group that determines the amino acid type. The " $\mathrm{R}$ " groups are referred to as the side chain and the other parts are called the main chain [1].

\subsection{The Nucleic Acids: Base, Backbone, Base Pair, Base Step}

Nucleic acids are sequences of nucleotides. A nucleotide consists of three parts: a five-carbon sugar, a nitrogen-containing ring structure called a base, and one, two, or three phosphate groups. Sugar and phosphorus are referred to as the backbone [1].

\subsection{The Interactions: Single, Bidentate and Complex Interaction}

As shown in Fig. 1, an interaction with one hydrogen bond between an amino acid and a nucleotide is called single interaction. An interaction with two or more hydrogen bonds with either a nucleotide or base paired nucleotides is known as a bidentate interaction. An interaction where an amino acid binds to more than one base step simultaneously is called a complex interaction. Our definition of the interactions is slightly different from Luscombe's definition [2]. Luscombe's definition considers the binding with the base part only, but we also consider the binding with the backbone part (that is, the sugar and phosphorus) [2]. Therefore, this study can show the difference in the binding propensities between the base, sugar and phosphorus.

\subsection{The Hydrogen Bonds: Donor, Acceptor}

A hydrogen bond is formed by three atoms: one hydrogen atom and two electronegative atoms (often $\mathrm{N}$ or $\mathrm{O}$ ). The hydrogen atom is covalently bound to one of the electronegative atoms, and is called a hydrogen bond donor. The other electronegative atom is known as the hydrogen bond acceptor. The two electronegative atoms may take up some of the electron density from the hydrogen atom. As a result, each electronegative atom carries partial negative charge and the hydrogen atom carries a partial positive charge. Consequently, the hydrogen atom and the hydrogen bond acceptor can then have attractive interactions. The strength of the hydrogen bond depends on the donor and acceptor as well as their environment. The bond energy usually ranges from 1 to $5 \mathrm{kcal} / \mathrm{mol}$. This energy is smaller than the covalent bond energy, but greater than the thermal energy $(0.6 \mathrm{kcal} / \mathrm{mol}$ at room temperature). Therefore, a hydrogen bond can provide a significant stabilizing force in macromolecules such as proteins and nucleic acids [3]. 


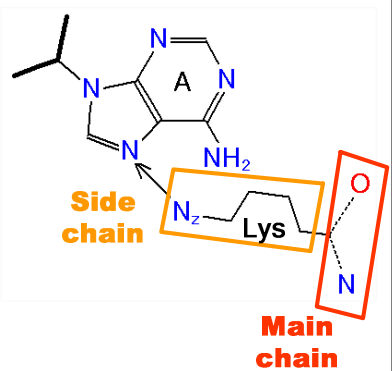

1. Single Interaction

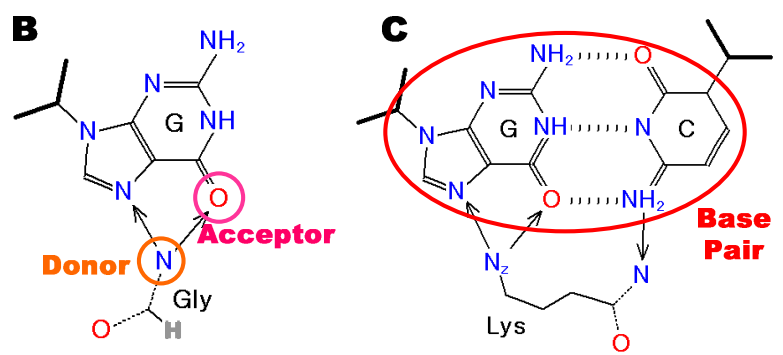

2. Bidentate Interaction
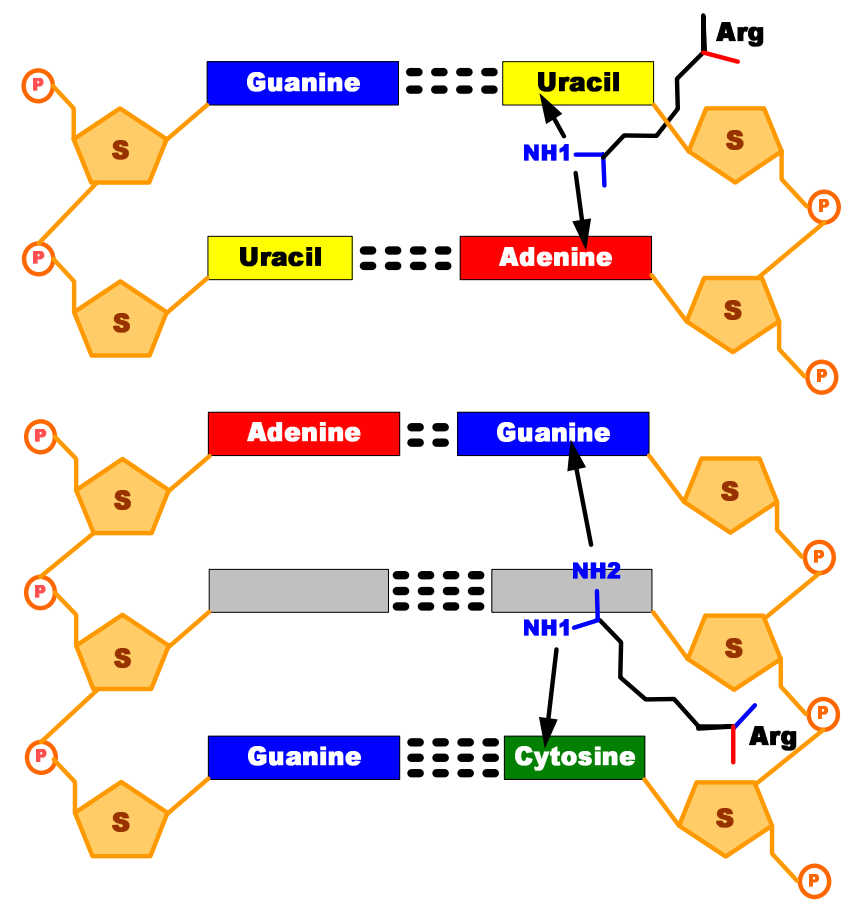

3. Complex Interaction

Fig. 1. Schematic diagram of three interactions 


\section{Framework for Analyzing Protein-RNA Binding Structures}

\subsection{Dataset}

The protein-RNA complex structures were obtained from the PDB database [4]. The complexes, which were determined by $\mathrm{X}$-ray crystallography with a resolution $\geq 3.0 \AA$, were selected. As of September 2002, there were 188 protein-RNA complexes in PDB and the number of complexes with a resolution of $3.0 \AA$ or better is 139 . We used PSIBLAST [5] for a similarity search on each of the protein and RNA sequences in these 139 protein-RNA complexes in order to eliminate the equivalent amino acids or nucleotides in homologous protein or RNA structures. 64 out of 139 protein-RNA complexes were left as the representative, non-homologous complexes after running the PSI-BLAST program with an E value of 0.001 and an identity value of $80 \%$ or below. We excluded 13 out of the 64 complexes that have no information of water molecules or are composed of artificial nucleotides. Therefore, the final data set was composed of 51 protein-RNA complexes. Table 1 shows the list of 51 protein-RNA complexes studied in our analysis.

Table 1. The 51 protein-RNA complexes in the data set

\begin{tabular}{ccccccccc}
\hline & \multicolumn{7}{c}{ PDB code } \\
\hline $1 \mathrm{~B} 23$ & $1 \mathrm{~B} 2 \mathrm{M}$ & $1 \mathrm{~B} 7 \mathrm{~F}$ & $1 \mathrm{C} 0 \mathrm{~A}$ & $1 \mathrm{C} 9 \mathrm{~S}$ & $1 \mathrm{CX} 0$ & $1 \mathrm{DFU}$ & $1 \mathrm{DI} 2$ & $1 \mathrm{DK} 1$ \\
$1 \mathrm{E} 7 \mathrm{X}$ & $1 \mathrm{EC6}$ & $1 \mathrm{EFW}$ & $1 \mathrm{~F} 7 \mathrm{U}$ & $1 \mathrm{~F} 8 \mathrm{~V}$ & $1 \mathrm{FEU}$ & $1 \mathrm{FFY}$ & $1 \mathrm{FXL}$ & $1 \mathrm{G} 59$ \\
$1 \mathrm{GAX}$ & $1 \mathrm{GTF}$ & $1 \mathrm{GTN}$ & $1 \mathrm{G} 2 \mathrm{E}$ & $1 \mathrm{H} 4 \mathrm{Q}$ & $1 \mathrm{H} 4 \mathrm{~S}$ & $1 \mathrm{HC} 8$ & $1 \mathrm{HDW}$ & $1 \mathrm{HE} 0$ \\
$1 \mathrm{HE} 6$ & $1 \mathrm{HQ} 1$ & $1 \mathrm{I} 6 \mathrm{U}$ & $1 \mathrm{IL} 2$ & $1 \mathrm{JBR}$ & $1 \mathrm{JBS}$ & $1 \mathrm{JID}$ & $1 \mathrm{~K} 8 \mathrm{~W}$ & $1 \mathrm{KNZ}$ \\
$1 \mathrm{KQ} 2$ & $1 \mathrm{~L} 9 \mathrm{~A}$ & $1 \mathrm{LNG}$ & $1 \mathrm{MMS}$ & $1 \mathrm{QF} 6$ & $1 \mathrm{QTQ}$ & $1 \mathrm{SER}$ & $1 \mathrm{URN}$ & $1 \mathrm{ZDH}$ \\
$1 \mathrm{ZDI}$ & $2 \mathrm{BBV}$ & $2 \mathrm{FMT}$ & $5 \mathrm{MSF}$ & $6 \mathrm{MSF}$ & $7 \mathrm{MSF}$ & & & \\
\hline
\end{tabular}

\subsection{Hydrogen Bonds}

The number of hydrogen bonds between the amino acids and nucleotides in the protein-RNA complexes was calculated using CLEAN, which is a program used for tidying Brookhaven files, and HBPLUS [6], which is a program to calculate the number of hydrogen bonds. The hydrogen bonds were identified by finding all proximal atom pairs that satisfy the given geometric criteria between the hydrogen bond donors (D) and acceptors (A). The positions of the hydrogen atoms $(\mathrm{H})$ were theoretically inferred from the surrounding atoms, because hydrogen atoms are invisible in purely $\mathrm{X}$ ray-derived structures. The criteria considered to form the hydrogen bonds for this study are: contacts with a maximum D-A distance of $3.9 \AA$, maximum H-A distance of $2.5 \AA$, and a minimum D-H-A angle and H-A-AA angle set to $90^{\circ}$, where AA is an acceptor antecedent.

All the hydrogen bonds were extracted from the HBPLUS output files. There were 1568 hydrogen bonds in the dataset. In order to compare the properties of a single interaction, a bidentate interaction and a complex interaction, separate experiments were conducted and the results were analyzed for the three classes of protein-RNA complexes: (1) single interaction, (2) bidentate interaction and (3) complex interaction. Figure 2 shows the sequence of classifying the three interaction types. 


\section{Algorithms}

This section describes the seven algorithms, which were performed in sequence on the data shown in Fig. 2. The terminologies used in the algorithms are explained in Sect. 2.

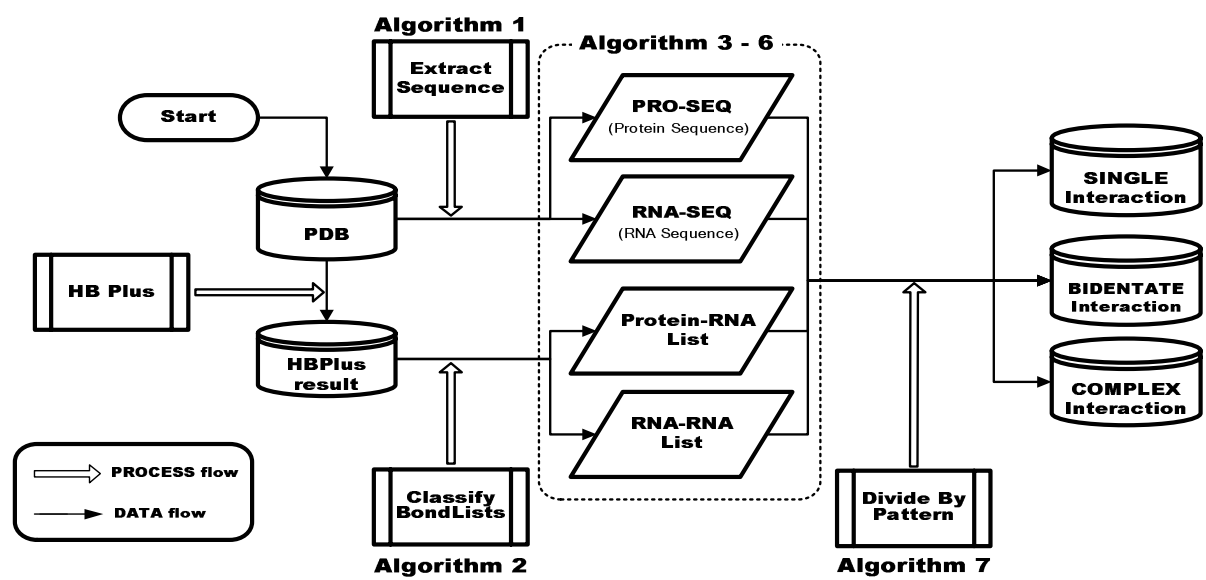

Fig. 2. Sequence of classifying three interaction types. The operation within a box labeled by an algorithm is explained in section 4. HBPLUS is explained in section 3.2.

Algorithm 1 constructs the PRO-SEQ and RNA-SEQ arrays to store the amino acid and RNA sequences, respectively. Algorithm 2 classifies hydrogen bonds into a P-RList (list of hydrogen bonds between the protein and RNA) and R-R-List (list of hydrogen bonds between RNA and RNA). Algorithm 3 investigates the internal hydrogen bond relations of the RNA and marks the result of the investigation in a linked-list. Algorithm 4 investigates the hydrogen bonds between the protein and RNA and marks the result of the investigation in a linked-list. Algorithm 5 analyzes whether a nucleotide is paired with another nucleotide. It returns true if the nucleotide is paired. Algorithm 6 classifies the type of each amino acid into unary, binary and multi-bond based on the number of hydrogen bonds between the amino acid and the RNA. It calls Get_Max_Distance() to calculate the distance between a nucleotide and its binding amino acid. Algorithm 7 classifies the protein-RNA interaction types into three categories. The three categories are single interactions, bidentate interactions and complex interactions, as was explained in Sect. 2.

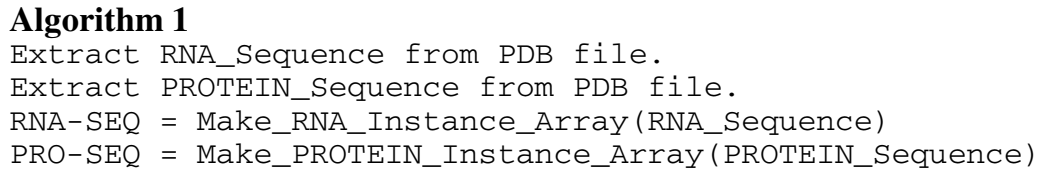



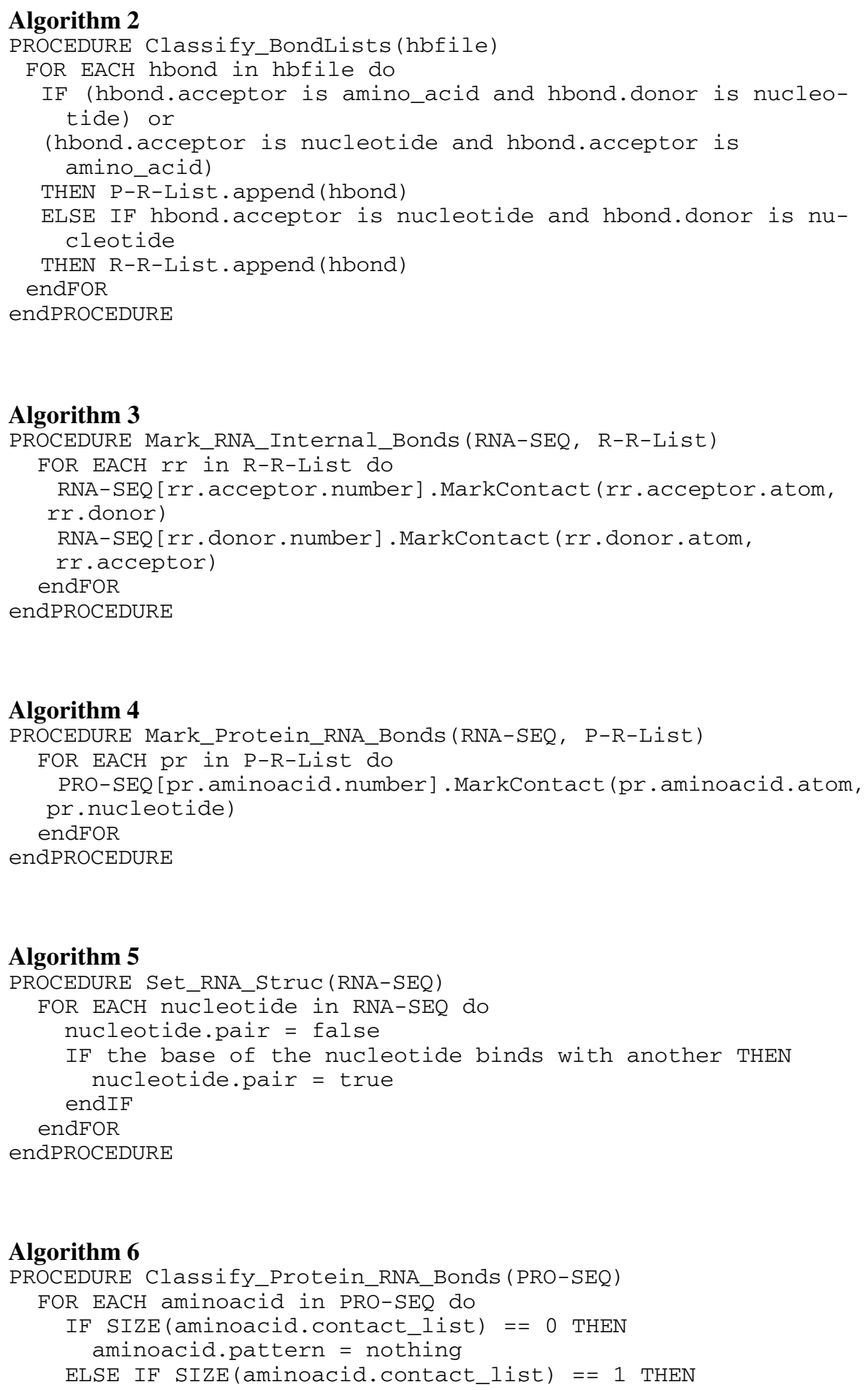

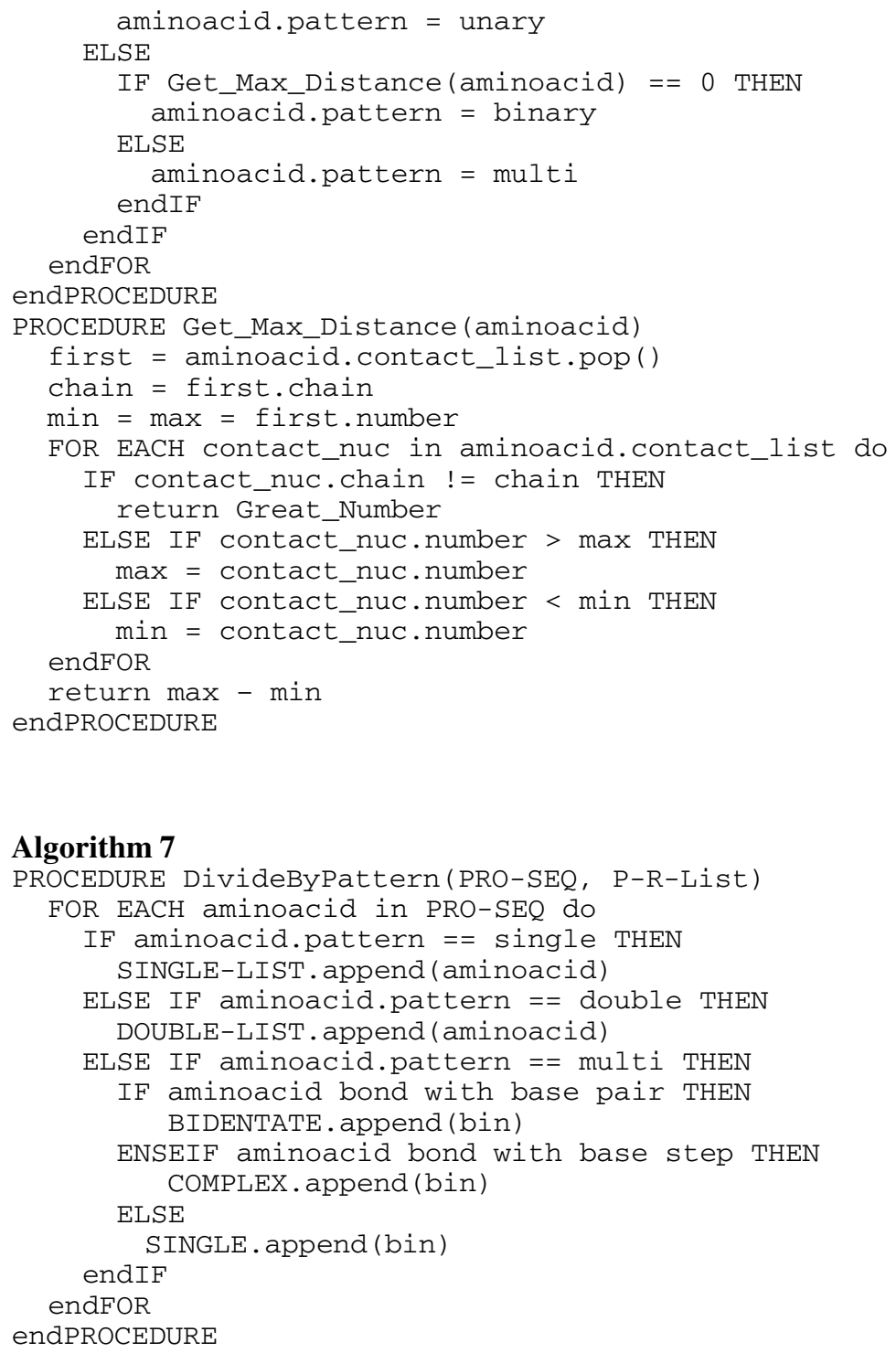

\section{Results and Discussion}

The protein-RNA interactions were computed for the 51 complexes. All interactions that occur repeatedly in the structurally related complexes were removed from the dataset in order to exclude any bias towards the proteins with multiple PDB entries. This filtering process resulted in a dataset of 1568 hydrogen bonds, which were the subject of this analysis. 


\subsection{Amino Acids}

Among the three interaction types, single interactions are the most abundant, as shown in Table 2. Amino acids involved in the bidentate interactions predominantly interact with bases. Table 3 shows the ranking of bindings of the amino acids with nucleotides in each interaction type. The dominant bindings are shown in bold style. For example, PHE-G S in the bold style denotes that PHE binds mainly to the sugar part of guanine but not to the other parts. No hydrophobic residues were found in the complex interactions. In particular, ALA, MET, PRO and VAL were found in single interactions only.

Table 2. The number of hydrogen bonds in the nucleotides for each interaction type

\begin{tabular}{lrrrr}
\hline & BASE & SUGAR & PHOSPHORUS & \multicolumn{1}{c}{ TOTAL } \\
\hline SINGLE & $464(46 \%)$ & $267(26 \%)$ & $281(28 \%)$ & $1012(65 \%)$ \\
BIDENTATE & $286(60 \%)$ & $142(30 \%)$ & $48(10 \%)$ & $476(30 \%)$ \\
COMPLEX & $16(20 \%)$ & $29(36 \%)$ & $35(44 \%)$ & $80(5 \%)$ \\
\hline TOTAL & $766(49 \%)$ & $438(28 \%)$ & $364(23 \%)$ & $1568(100 \%)$ \\
\hline
\end{tabular}

Table 3. The top ten hydrogen bonds frequently participating in each interaction type. The bonds in the bold style represent they occur frequently

\begin{tabular}{cccccccc}
\hline rank & TOTAL & rank & SINGLE & rank & BIDENTATE & rank & COMPLEX \\
\hline 1 & GLU - G B 89 & $\mathbf{1}$ & THR - G B 37 & $\mathbf{1}$ & GLU - G B 74 & 1 & ARG - C P 10 \\
2 & LYS - A B 69 & $\mathbf{2}$ & PHE - G S 29 & 2 & LYS - A B 41 & 2 & ARG - U P 7 \\
3 & ASP - G B 60 & 2 & ARG - U B 29 & 3 & THR - A B 36 & 3 & ARG - A P 5 \\
4 & THR - A B 58 & 4 & LYS - A B 28 & $\mathbf{4}$ & ASP - G B 31 & 3 & ASP - G S 5 \\
5 & ARG - C P 42 & 4 & LYS - C P 28 & 5 & ARG - C B 23 & 3 & ARG - C S 5 \\
6 & SER - G S 39 & 6 & ASP - G B 27 & 6 & ASN - U S 18 & 6 & ARG - U S 4 \\
7 & THR - G B 38 & 7 & SER - A B 25 & 7 & SER - G S 13 & 7 & ARG - G P 3 \\
7 & ARG - U B 38 & 7 & SER - G S 25 & $\mathbf{8}$ & HIS - G S 10 & 7 & HIS - G S 3 \\
9 & ARG - C B 35 & 7 & LYS - U P 25 & 8 & SER - U S 10 & 7 & LYS - G B 3 \\
10 & PHE - G S 31 & 10 & LYS - A P 24 & 10 & THR - A S 9 & 7 & LYS - C P 3 \\
10 & LYS - C P 31 & 10 & LYS - G B 24 & 10 & ARG - U B 9 & & \\
& & 10 & ARG - C P 24 & 10 & ARG - U S 9 & & \\
\hline
\end{tabular}

A: adenine, G: guanine, $\mathrm{C}$ : cytosine, $\mathrm{U}$ : uracil

B: base of nucleotide, S: sugar of nucleotide, P: phosphorus of nucleotide 


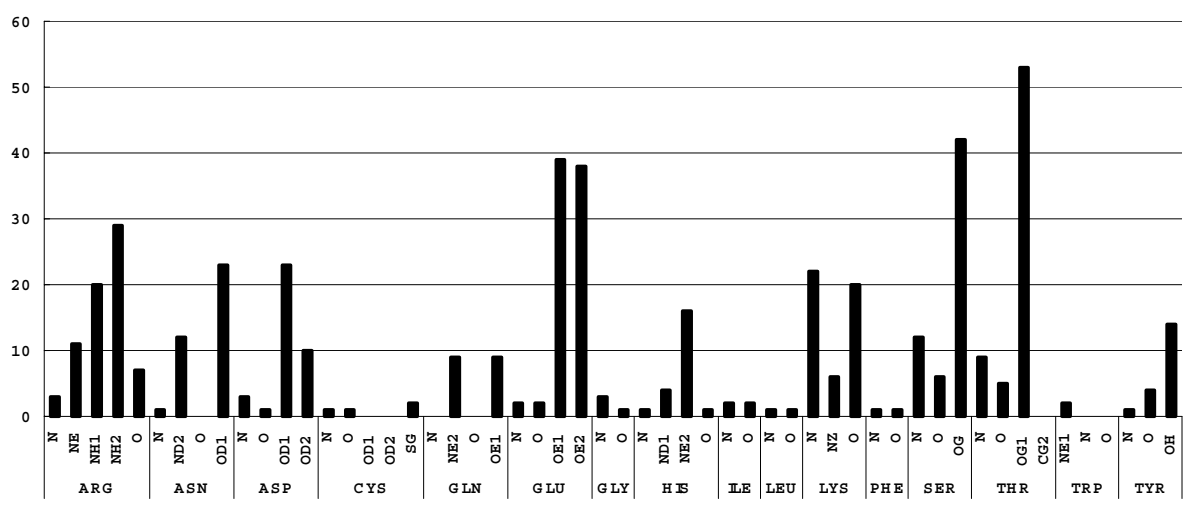

Fig. 3. The total number of hydrogen bonds of each amino acid, involved in the bidentate interactions with nucleotides. ALA, MET, PRO and VAL are not shown here because they were not involved in any bidentate interaction

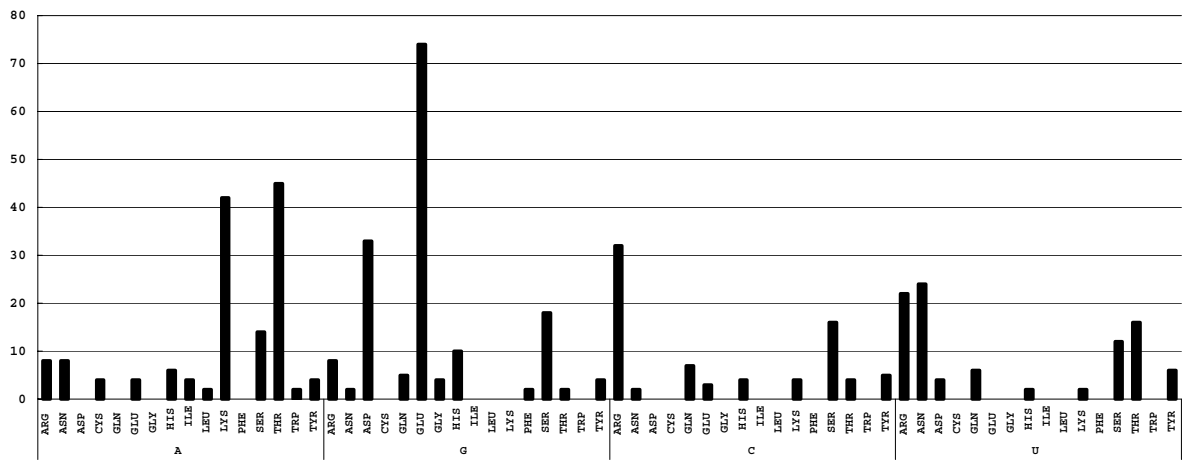

Fig. 4. The total number of hydrogen bonds of each nucleotide, involved in the bidentate interactions with amino acids

The number of hydrogen bonds in the amino acids is as follows. ARG (306), LYS (257), SER (164), THR (151), GLU (136), ASN (125), ASP (116), GLN (61), TYR (59) and GLY (40). But the ordering changes when the hydrogen bonds are classified based on whether they are found in the base, sugar or phosphorus of a nucleotide. LYS and THR frequently participate in the bonds with a base but not with sugar. In contrast, ARG and SER frequently participate in bonding with the backbone but not with the base. THR showed a significant dissimilarity from SER. THR is 2nd most common in binding to a base but the 10th in binding to a sugar. In contrast, SER is the 7 th most common in binding to a base but 2 nd in binding to a sugar. Fig. 3 shows the number of hydrogen bonds of each amino acid, involved in the bidentate interactions.

From an atomic point of view, the LYS NH binds to the phosphorus of the nucleotides 87 times and is the most frequent participator. Next are the ARG NH2phosphorus and the THR O-base, which bind 58 times. The order of the others is as 
follows: ASP OD1-base (55 times), GLU OE2-base (51), SER OG-sugar (51), LYS NZ-base (49), SER OG-base (49), ARG NH2-base (45) and GLU OE1-base (45). This study made two interesting observations. The first observation was that 82 LYS NZ-phosphorus bonds out of a total 87 were observed in the single interactions. However, the THR OG1-base bond participates mainly in bidentate interactions. The second observation was that even though the THR O and OG1 consist of the same amino acids and bind to the same nucleotide part, this study found that the $\mathrm{O}$ is mainly involved in single interactions and the OG1 is involved in the bidentate interactions. This is partly, but not entirely, due to the difference in the side chain and the main chain. Both the THR OG1 and OG2 are located in the amino acid side chain. However, OG2 never binds while OG1 binds 46 times. Different amino acids showed different binding propensities depending on the interaction types. GLU and HIS participate mainly in bidentate interactions. In particular, GLU binds to a base frequently because GLU OE1 and OE2 have a strong binding propensity.

\subsection{Nucleotide}

On average, hydrogen bonds prefer the bases (49\%) than the sugar (28\%) and phosphorus (23\%) of a nucleotide, but there is little difference in preference between the bases and the backbone (Table 2). The specific binding propensities change according to interaction types. In bidentate interactions, the hydrogen-bonding rate of the base increases to $60 \%$ and that of phosphorus decreases to $10 \%$. In contrast, in complex interactions, the hydrogen-bonding rate of the base decreases to $20 \%$ and that of phosphorus increases to $44 \%$. This difference can be explained by the structure. In complex interactions, an amino acid binds to at least two base pairs, which is a stem. Thus, an amino acid held within a stem has a great chance to bind to the backbone.

All the nucleotides bind frequently to ARG and LYS. However, guanine binds predominantly with GLU and ASP, which have an acidic side chain group, and adenine binds frequently to THR. This is because GLU OE1, OE2 and THR OG1 bind preferentially to the base in bidentate interactions. Figure 4 shows the number of hydrogen bonds of each nucleotide, which are involved in bidentate interactions.

\section{Conclusion}

Structural analysis of the protein-RNA complexes is labor-intensive yet it provides insight into the interaction patterns between a protein and RNA. The protein-RNA binding structures are studied either individually or on a small-scale. However, manual analysis of the protein-RNA binding structures is becoming increasingly challenging as the complexity and number of protein-RNA complexes increase. This study developed a set of algorithms for automatically analyzing the hydrogen bonds in the protein-RNA binding structures and for identifying the interaction patterns between a protein and RNA. The algorithms were used for analyzing 1568 hydrogen bonds in 51 protein-RNA complexes, which are the most representative set of protein-RNA complexes known today. This is the first computational approach for analyzing the structures of a large set of protein-RNA complexes. The interaction patterns discovered 
from this analysis will assist in the understanding of how proteins interact with RNA with specificity and to predict the structure of the RNA binding protein as well as the structure of the protein binding RNA.

\section{References}

1. Lesk, A.M.: Introduction to Protein Architecture (2nd Edition) Oxford University Press (2001)

2. Luscombe, N.M., Laskowski, R.A., Thornton, J.M.: Amino acid-base interactions: a threedimensional analysis of protein-DNA interactions at an atomic level. Nucleic Acids Research 29 (2001) 2860-2874

3. Web-Book Home Page http: / / www. web-books. com/

4. Berman, H.M., Westbrook, J., Feng, Z., Gilliland, G., Bhat, T.N., Weissig, H., Shindyalov, I.N., Bourne, P.E.: The Protein Data Bank. Nucleic Acids Research 28 (2000) 235-242

5. Altschul, S.F., Madden, T.L., Schaffer, A.A., Zhang, J., Zhang, Z., Miller, W., Lipman, D.J.: Gapped BLAST and PSI-BLAST: a new generation of protein database search programs. Nucleic Acids Research 25 (1997) 3389-3402

6. McDonald, I.K. Thornton, J.M.: Satisfying Hydrogen Bonding Potential in Proteins. J. Mol. Biol. 238 (1994) 777-793 